\title{
Variations of soil resistivity values due to grounding system installations with natural enhancement material mixtures
}

\begin{abstract}
Soil resistivity is a crucial parameter to be taken into consideration in designing any electrical grounding system, as the understanding towards its changes over time and soil properties is crucial to ensure transients and fault currents to be able to be dispersed to the earth in shortest time and shortest path possible. National and international standards stated that there is necessity to measure soil resistivity of a site before any grounding system installation could be carried out at that site. Steps and tests needed to be done in finding a suitable site for grounding system installations with Natural Enhancement Material Mixtures were discussed in this work. Three sites were taken into consideration, which only one to be selected, based on soil resistivity assessments that were carried out. The effectiveness of the Natural Enhancement Material Mixtures grounding systems installed at the selected site were also monitored for the duration of one year, by measuring the soil resistivity of the site once in three months. Note that all the sites are located in Universiti of Putra Malaysia, Serdang, Selangor, Malaysia. It is shown that there is general relationship between the soil resistivity values and climate change in Malaysia throughout a year of measurement duration. Also, by installing grounding systems with Natural Enhancement Material Mixtures, the soil resistivity values were slightly reduced over a period of one year.
\end{abstract}

